\title{
Communications
}

\section{Examens de spécialiste \\ Examen de spécialiste en vue de l'obtention du titre de spécialiste en cardiologie}

Examen écrit: European Exam in General Cardiology (EEGC)

Date: mercredi 12 juin 2019

Lieu: à Lausanne et à Zurich, $1 \times$ le matin / $1 \times$ l'après-midi

Veuillez nous indiquer impérativement quel local ET quelle heure du jour vous préférez.

Important: Sur l'inscription le nom/prénom de la candidate / du candidat doit être indiqué exactement comme écrit dans le passeport / sur la carte d'identité - en cas de différences, l'admission à l'examen ne sera pas possible.

\section{Délai d'inscription: 30 novembre 2018}

Vous trouverez de plus amples informations sur le site web de l'ISFM www.siwf.ch $\rightarrow$ Domaines spécialisés $\rightarrow$ Titres de spécialiste et formations approfondies (formation postgraduée) $\rightarrow$ Cardiologie
Examen de spécialiste en vue de l'obtention du titre de spécialiste en cardiologie

Examen oral

Date: jeudi 24 octobre 2019

Lieu: Hôpital Universitaire, Bâle (en allemand)

Hôpitaux Universitaires de Genève (en français)

\section{Délai d'inscription: 31 août 2019}

Vous trouverez de plus amples informations sur le site web de l'ISFM www.siwf.ch $\rightarrow$ Domaines spécialisés $\rightarrow$ Titres de spécialiste et formations approfondies (formation postgraduée) $\rightarrow$ Cardiologie
Examen de spécialiste en vue de
l'obtention du titre de spécialiste en
médecine physique et réadaptation

Examen oral

Date: le vendredi 31 mai 2019

Lieu: Stadtspital Triemli, Birmensdorferstrasse 497, 8063 Zurich

Délai d'inscription: 29 mars 2019

Vous trouverez de plus amples informations sur le site web de l'ISFM www.siwf.ch $\rightarrow$ Domaines spécialisés $\rightarrow$ Titres de spécialiste et formations approfondies (formation postgraduée) $\rightarrow$ médecine physique et réadaptation

\section{Sujet d'actualité en ligne} www.bullmed.ch/fr/tour-dhorizon

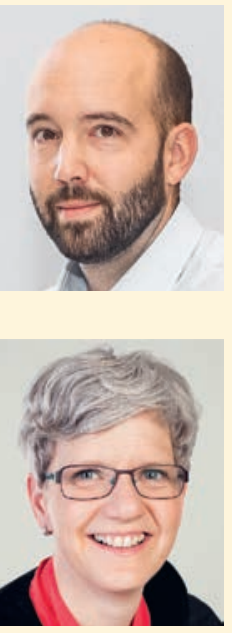

Interview du Dr sc. Dominik Glinz, Institut d'épidémiologie clinique et de biostatistique de l'Université de Bâle

\section{Il est possible d'améliorer l'usage des antibiotiques}

Une étude menée en Suisse à l'échelle nationale révèle qu'il est tout à fait possible d'améliorer l'usage des antibiotiques dans les cabinets des médecins de famille.

Sandra Ziegler, présidente de la direction de EMH Editions médicales suisses SA

\section{«La qualité est notre plus grande force»}

Nouvelle direction pour les Editions médicales suisses. 